\title{
La red de apoyo: Una herramienta esencial para el desempeño escolar del estudiante
}

\section{The support network: An essential tool for student school performance}

\author{
VILLARREAL-SOTO, Blanca Margarita†*, CEPEDA-GONZÁLEZ, María Cristina, SANCHEZ- \\ RIVERA, Lilia, ESPERICUETA-MEDINA, Marta Nieves y GARCÍA-OVALLE, Samanta Irasema
}

Universidad Autónoma de Coahuila, Fac. de Ciencia Educación y Humanidades

ID $1^{\text {er }}$ Autor: Blanca Margarita, Villarreal-Soto / ORC ID: 0000-0001-9314-8001, Researcher ID Thomson: 2357-2018, CVU CONACYT ID: 947979

ID $1^{\text {er }}$ Coautor: María Cristina, Cepeda-González / ORC ID: 0000-0003-0676-2412, Researcher ID Thomson: 1651-2018, CVU CONACYT ID: 567204

ID $2^{\text {do }}$ Coautor: Lilia, Sanchez-Rivera / ORC ID: 0000-0001-9468-2599, Researcher ID Thomson: 1404-2018, CVU CONACYT ID: 613195

ID $3^{\text {er }}$ Coautor: Marta Nieves, Espericueta-Medina / ORC ID: 0000-0002-4924-4332, Researcher ID Thomson: T-15002018, CVU CONACYT ID: 372705

ID $4^{\text {to }}$ Coautor: Samanta Irasema, García-Ovalle / ORC ID: 0000-0001-5409-2148, Researcher ID Thomson: T-ABI-59502020, CVU CONACYT ID: 1098122

DOI: $10.35429 / J U M .2020 .11 .4 .27 .33$

Recibido 12 de Abril, 2020; Aceptado 30 de Junio, 2020

\section{Resumen}

Revisando diversos autores se encontró que para que un estudiante pueda desempeñarse mejor en el ámbito educativo necesita apoyo tanto de los docentes como de su familia, es por ello que surge la interrogante de investigación. ¿Cómo interviene la orientación docente en la estructura familiar? El presente trabajo es de tipo perimental, se elaboró un instrumento constituidos por 66 variables de las cuales 6 son variables signalíticas y el resto constituyen el fenómeno a estudiar, el instrumentó se aplicó a 144 sujetos. La base de datos obtenida se trabajó en los niveles estadísticos de caracterización, correlación y análisis integracional procesándose en el programa Statistica y Spss. Entre los principales resultados se encontró que la familia es un factor importante en la orientación docente del estudiante ya que si el estudiante tiene relaciones familiares cercanas, donde ellos se sientan queridos y aceptados tienen un efecto positivo en el desempeño escolar de los estudiantes.

Familia, Estructura, Orientación, Docente, Estudiantes

\begin{abstract}
Reviewing various authors it was found that in order for a student to perform better in the educational field needs support from both teachers and their family, that is why the research question arises. How does teaching guidance intervene in the family structure? This work is of a perimental type, an instrument consisting of 66 variables of which 6 are signatory variables was developed and the rest constitute the phenomenon to be studied, the instrument was applied to 144 subjects. The database obtained was worked on the statistical levels of characterization, correlation and integrational analysis processed in the Statistica and Spss program. Among the main results was that the family is an important factor in the student's teaching orientation since if the student has close family relationships, where they feel loved and accepted they have a positive effect on the student's school performance.
\end{abstract}

Family, Structure, Orientation, Teacher, Students

Citación: VILLARREAL-SOTO, Blanca Margarita, CEPEDA-GONZÁLEZ, María Cristina, SANCHEZ-RIVERA, Lilia, ESPERICUETA-MEDINA, Marta Nieves y GARCÍA-OVALLE, Samanta Irasema. La red de apoyo: Una herramienta esencial para el desempeño escolar del estudiante. Revista de Gestión Universitaria. 2020. 4-11: 27-33

\footnotetext{
* Correspondencia del Autor (Correo electrónico: mnieves@ uadec.edu.mx)

$\dagger$ Investigador contribuyendo como primer autor.
} 


\section{Introducción}

Involucrar a los padres en la orientación docentes es importante ya que logra concientizar en ellos, lo importante que es la participación y apoyo de la familia en el proceso educativo de los alumnos de primer año de secundaria.

Escuela y familia han de compartir inquietudes, intercambiar informaciones $y$ pensamientos sobre la educación, la escuela, los hijos. Y ayuda a establecer pactos y acuerdos sobre ciertas actuaciones hacia el niño/a. La familia tiene que aplicar los acuerdos tomados e intentar traspasar los conocimientos escolares a la vida diaria. Y la escuela debe alcanzar en cada niño/a los objetivos acordados o propuestos y traspasar y aplicar los conocimientos familiares y cotidianos a la vida escolar de manera que se consiga esta interrelación y unión entre la educación formal y no formal y ese apoyo y eficacia esperada.

En la actualidad, existe una gran preocupación por el poco interés que demuestran los padres de familia en el rendimiento escolar de sus hijos, las causas son múltiples, el trabajo de los padres durante todo el día o la desintegración familiar, son algunos de los factores que influyen en este nulo apoyo educativo de los padres, dejando toda la responsabilidad a las instituciones educativas y por lo tanto a los maestros. Es por ello, que se realizó esta investigación para comprobar que la familia es un factor importante en la orientación docente.

\section{Marco teórico}

(Santodomingo, 2016), La Orientación Docente es el rol que puede llegar a desarrollar cualquier profesor de un centro educativo - deseablemente todos los docentes y a ser posible de manera coordinada con los demás profesores y con los orientadores - a través del cual proporcionará un apoyo a todo su alumnado para que pueda tomar decisiones sobre su itinerario formativo y su futuro profesional en las mejores condiciones posibles. Como menciona el autor la orientación docente permite que el estudiante tome decisiones o se guie en el ámbito formativo al igual que mejorando en él.
(Rodriguez, 1991) Orientar es, fundamentalmente, guiar, conducir, indicar de manera procesual para ayudar a las personas a conocerse a sí mismo y al mundo que los rodea; es auxiliar a un individuo a clarificar la esencia de su vida, a comprender que él es una unidad con significado capaz de y con derecho a usar de su libertad, de su dignidad personal dentro de un clima de igualdad de oportunidades y actuando en calidad de ciudadano responsable tanto en su actividad laboral como en su tiempo libre.

(Maher \& Forman, 1987) La orientación "es una aportación directa de información cara a cara, de asesoramiento o guía por parte del orientador a un grupo estudiantil o a un alumno individual". Los autores mencionan que la orientación docente, como anteriormente dicho, es una guía, pero también lo identifica con otra palabra que es asesoramiento ya que ayuda al estudiante que aspectos debe, mejorar y que aspectos debe fortalecer.

(Slideshare, 2012) La estructura familiar es un conjunto en interacción, organizado de manera estable y estrecha en función de necesidades básicas con una historia y un código propio que le otorga singularidad. Como menciona la página y como menciona el autor, la estructura familiar es un grupo donde las personas se relacionan, interactúan entre ellas, se organizan, etc. La estructura familiar permite además que las personas se desenvuelvan y puedan cumplir sus necesidades básicas.

(Silva., 2011), La estructura familiar es el soporte de todos los rasgos que caracterizan a una familia, le da identidad y distingue a una de otra. Esta identidad debe tener solidez y flexibilidad de límites. Como menciona la autora anteriormente la estructura familiar significa por ciertas características que la distinguen de una de otra por ejemplo sus hábitos, costumbres, tradiciones, etc. Es por ello por lo que ciertas familias tienen distintos nombres ya que se con forma de forma diferente

(Solórzano, 2020) La Estructura familiar es cuando la familia está organizada según el orden jerárquico en que se disponen sus miembros, donde cada posición le confiere obligaciones y prerrogativas delimitadas por reglas concretas las cuales contribuyen a vincular el funcionamiento de la familia con los fines del grupo familiar. 
(Ortega, 2007) La estructura familiar no es un fenómeno estático, sino un proceso dinámico que varía según el momento histórico en que se encuentre la familia, la etapa del ciclo vital por la que transite o determinados acontecimientos vitales. Asimismo, las familias evolucionan y cambian en la medida en que lo hace la sociedad, y uno de los cambios sociales que más ha influido en la estructura familiar ha sido el económico.

Como menciona el autor la estructura familiar no es algo estático es algo que va evolucionando dependiendo del tiempo o el contexto donde se está desarrollando, también a través de esto también se van cambiando los valores, tradiciones o hábitos que han tenido las familias porque se van acomodando con los cambios sociales que han influido, pero no significa que vayan desapareciendo valores si no que se van ordenando con la importancia que tienen ciertos valores que antes no se creían importantes o ciertas tradiciones que tampoco se creen importantes.

\section{Metodología}

El presente estudio es de tipo perimental, transveral y cuantitativa, se elabora un instrumento para medir la frecuencia relativa de los datos que se va a trabajar.

La estructura de operación se centra en la elaboración de un planteamiento de investigación, el diseño de una metodología de trabajo y la operacionalización de resultados para la formulación de propuestas de intervención.

El tema de esta investigación es La Orientación Docente y el contraste de esta es La Estructura Familiar. Se investiga cómo en la preparatoria 1 llega a ser la orientación docente tomando en cuenta cómo es la estructura familiar del estudiante.

Con el objetivo de dar explicación a los ejes de investigación los estadísticos se llevarán a cabo, a través de diferentes programas estadísticos: frecuencias y porcentajes, correlación, caracterización e integración.

\section{Resultados}

\section{Frecuencias y porcentajes}

El $42.36 \%$ de los sujetos encuestados son del género masculino y el $57.64 \%$ son del género femenino; con edades que oscilan de 14 a 17años. El 14.58\% de los encuestados les gusta leer, el $25.69 \%$ les gusta ver televisión, el $29.17 \%$ les gusta realizar otras actividades y al $30.56 \%$ les gusta escuchar música. Asimismo, el $8.33 \%$ de los encuestados les gusta otras materias de las que se presentaron como opciones, al $10.42 \%$ les gusta la materia física, al $15.97 \%$ les gusta la materia de informática, al $17.36 \%$ les gusta la materia de inglés, al $20.78 \%$ les gusta la materia de matemáticas y al $27.78 \%$ les gusta la materia de TLR. Otro dato importante es que los estudiantes son de primero, segundo, tercero, cuarto semestre. Por último, el $9.72 \%$ de los encuestados tiene más de 4 hermanos, el $15.97 \%$ tiene un hermano, $18.75 \%$ tiene 4 hermanos y el $55.56 \%$ tiene entre 2 y 3 hermanos.

\section{Caracterización}

En este apartado se observan los resultados arrojados de la orientación docente y la estructura familiar al aplicar las medidas de tendencias central y las medidas de dispersión.

\section{La orientación docente}

Las 5 variables atípicas relevantes son: el grado de la relación que tiene el profesor con el estudiante $(\bar{x}=6.10)$, la medida de la conducta del profesor en el ámbito escolar $(\bar{x}=6.16)$, el grado de conducción que realiza el profesor hacia el aprendizaje ( $\bar{x}=5.97)$, la medida de cumplimiento del estudiante con sus obligaciones $(\bar{x}=6.15)$ y el grado de cumplimiento de las responsabilidades del profesor $(\overline{\mathrm{x}}=6.07)$.

Por lo que se puede inferir que para que una orientación docente sea de calidad se necesita tanto responsabilidad del estudiante como la del profesor, también un comportamiento eficaz del profesor y más que nada una motivación por parte de los involucrados para un trabajo efectivo. 
Las 3 variables atípicas irrelevantes son el grado de la relación que tiene el profesor con el estudiante $(\bar{x}=5.32)$, el nivel de obstáculo que tiene el estudiante para poder aprender $(\overline{\mathrm{x}}=5.20)$ y el grado de intervención que realiza el profesor al estudiante para trabajar con su personalidad $(\bar{x}=5.38)$.

Por lo que se puede inferir que para que una orientación docente se ha de calidad tiene que tener un apoyo hacia el alumno, el conocimiento técnico, el conocimiento científico, atención del profesor, la intervención continua, la compresión del estudiante, la revisión de profesor, la planificación de los contenidos, la ayuda individualizada, la autorrealización personal, el tomar propias decisiones, facilitar el aprendizaje, la personalidad del educando, el estudio del problema, el estudio del problema, la creatividad del estudiante, la enseñanza del estudiante, la intervención técnica, el ambiente al enseñar, el progreso el estudiante, el desarrollo escolar, la igualdad de oportunidades y las herramientas didácticas que utiliza el profesor.

\section{La estructura familiar}

Las 5 variables atípicas relevantes son: la medida de formación educativa del estudiante por parte de su familia $(\bar{x}=6.31)$, el nivel de ayuda que da la familia para lograr lo que se propone el estudiante $(\overline{\mathrm{x}}=6.18)$, el nivel de protección que siente el estudiante cuando esta con su familia $(\overline{\mathrm{x}}=6.31)$, el grado de cuidado que los padres le dan al estudiante $(\overline{\mathrm{x}}=6.24)$ y el nivel de amor que siente el estudiante por su familia $(\overline{\mathrm{x}}=6.41)$.Por lo que se puede inferir que lo más relevante en la estructura familiar es el desarrollo educativo que se le da al estudiante, el apoyo familiar, la seguridad del estudiante, la crianza del estudiante y el amor familiar.

La única variable atípica irrelevante es: la medida de los intereses que tienen en común el estudiante con sus familiares $(\overline{\mathrm{x}}=5.14)$. Por lo que se puede inferir que lo menos importante en la estructura familiar son los intereses que tiene la familia.

\section{Correlación}

Se presenta la correlación por rangos de Spearman en el cual se procesaron 60 variables con capacidad predictiva de los coeficientes de correlación con 144 sujetos con un nivel de significancia de $\mathrm{p} \leq \mathrm{a} 0.001$, el análisis se procesó correlacionando los ejes: Orientación Docente y Estructura Familiar, el cual se realizó en el programa estadístico SPSS.

\section{La orientación docente y la estructura familiar}

En este apartado se presenta la lectura de intraeje de las 60 variables de la orientación docente con la estructura familiar. El nivel de ayuda del profesor al estudiante a comprender los contenidos vistos en clase tiene relación positiva con la medida de los intereses que tienen en común el estudiante con sus familiares $\left(\mathrm{r}_{\mathrm{s}}=0.82\right)$, también con el nivel de orden que tiene la familia para realizar diferentes tareas $\left(r_{s}=0.81\right)$, con el grado de cualidades que caracterizan a la familia del estudiante $\left(r_{s}=0.86\right)$, con el grado de relación que tiene el estudiante con su familia $\left(\mathrm{r}_{\mathrm{s}}=0.83\right)$, con la medida de parecido físico que tiene el estudiante con sus familiares $\left(r_{s}=0.85\right)$, con el nivel de los valores compartidos por el estudiante con la familia $\left(\mathrm{r}_{\mathrm{s}}=0.85\right)$, con el grado de tradiciones que sigue la familia del estudiante $\left(\mathrm{r}_{\mathrm{s}}=0.80\right)$, con la medida de formación educativa del estudiante por parte de su familia $\left(\mathrm{r}_{\mathrm{s}}=0.82\right)$, con el grado de la economía que tiene la familia del estudiante $\left(\mathrm{r}_{\mathrm{s}}=0.82\right)$, con el nivel del lugar donde el estudiante vive con su familia $\left(r_{s}=0.86\right)$, con el grado del rol que interpreta cada integrante de la familia del estudiante $\left(r_{\mathrm{s}}=0.88\right)$, con el nivel de necesidades vitales que la familia cumple para contribuir a la supervivencia del estudiante $\left(\mathrm{r}_{\mathrm{s}}=0.86\right)$, con el grado de unión que tienen los padres del estudiante $\left(r_{s}=0.85\right)$, con el grado de ayuda que los hermanos le brindan al estudiante $\left(\mathrm{r}_{\mathrm{s}}=0.88\right)$, con el grado de parientes de distintas generaciones que son cercanos al estudiante $\left(r_{s}=0.84\right)$, con el nivel de relación que tiene el estudiante con su familia $\left(r_{s}=0.71\right)$, con grado de crecimiento personal que tiene el estudiante $\left(\mathrm{r}_{\mathrm{s}}=0.85\right)$, con el nivel de ayuda que da la familia para lograr lo que se propone el estudiante $\left(\mathrm{r}_{\mathrm{s}}=0.79\right)$, con el grado de normas que pone tu familia para comportante $\left(\mathrm{r}_{\mathrm{s}}=0.88\right)$, con el nivel de protección que siente el estudiante cuando esta con su familia $\left(r_{s}=0.86\right)$, con el grado de cuidado que los padres le dan al estudiante $\left(\mathrm{r}_{\mathrm{s}}=0.86\right)$. 
Por lo que se infiere para que el estudiante tenga un desenvolvimiento certero en la función académica, para que se tenga un nivel aceptable, debe tener una relación estable con la familia, buena comunicación al igual que recibir apoyo moral, emocional de ellos, pero al igual el docente debe apoyar al estudiante ofreciéndole su disponibilidad, paciencia, tolerancia, carácter.

\section{Integración}

Del total de las 60 variables se obtuvo una estructura de 2 factores a través de componentes principales y rotación varimax normalizada, con 48.3 variables que explican el $80.75 \%$ de la varianza común en el estudio.

\section{Factor 1. "La base del estudiante"}

El nivel de ayuda del profesor al estudiante a comprender los contenidos vistos en clase (.604), el grado de información que obtiene el estudiante por medio del estudio (.530), el grado de conocimientos que obtiene el estudiante por medio de la práctica (.749), la medida de tiempo que el profesor le dedica al estudiante para ayudarle con sus dudas en clase (.493), el grado de participación constante del profesor para ayudar al alumno (.584), el nivel de entendimiento del estudiante sobre los contenidos académicos (.581), el grado de la relación que tiene el profesor con el estudiante (.405), el grado de interés que el alumno tiene sobre una clase (.675), la medida de la conducta del profesor en el ámbito escolar (.607), la medida de la evaluación que realiza el profesor para medir el aprendizaje del alumno (.589), el grado de conducción que realiza el profesor hacia el aprendizaje (.548), la medida del plan que tiene el profesor para estructurar los contenidos académicos (.612), el nivel de apoyo que da el profesor a cada estudiante (.653), el nivel de realización de las metas planteadas por el estudiante (.668), la medida de la decisión que toma el estudiante para resolver un determinado problema (.647), el nivel de obstáculo que tiene el estudiante para poder aprender (.460), el nivel de simplificación del aprendizaje por parte del profesor (.550), el nivel del carácter del profesor (.439), la medida del análisis del problema de aprendizaje del estudiante (.527 La medida del análisis del problema de aprendizaje del estudiante (.548), el nivel de facilidad que tiene el estudiante para generar nuevas ideas sobre las temáticas vistas en clase (.567).
Por lo que se infiere que para que el estudiante tenga éxito en la educación necesita apoyo tanto de los docentes y de la familia, necesita sentirse en confianza, al igual que aceptado y querido para sentirse en un ambiente de comodidad para poder desempeñarse mejor.

Factor 2. "El camino para alcanzar el
aprendizaje"

El nivel de ayuda del profesor al estudiante a comprender los contenidos vistos en clase (.656), el grado de información que obtiene el estudiante por medio del estudio (.689), el grado de conocimientos que obtiene el estudiante por medio de la práctica (.545), la medida de tiempo que el profesor le dedica al estudiante para ayudarle con sus dudas en clase (.748), el grado de participación constante del profesor para ayudar al alumno (.676), el nivel de entendimiento del estudiante sobre los contenidos académicos (.694), el grado de la relación que tiene el profesor con el estudiante (.774), el grado de interés que el alumno tiene sobre una clase (.582), la medida de la conducta del profesor en el ámbito escolar (.626), la medida de la evaluación que realiza el profesor para medir el aprendizaje del alumno (.708), el grado de conducción que realiza el profesor hacia el aprendizaje (.696), la medida del plan que tiene el profesor para estructurar los contenidos académicos (.695), el nivel de apoyo que da el profesor a cada estudiante (.625), la medida del desarrollo del potencial del estudiante (.635), la medida de la decisión que toma el estudiante para resolver un determinado problema (.627), el nivel de obstáculo que tiene el estudiante para poder aprender (.554), el nivel de simplificación del aprendizaje por parte del profesor (.698), el nivel del carácter del profesor (.726), la medida del análisis del problema de aprendizaje del estudiante (.742), el nivel de facilidad que tiene el estudiante para generar nuevas ideas sobre las temáticas vistas en clase (.703), el grado de enseñanza de calidad que recibe el estudiante por parte del profesor (.720), la medida de la actuación del profesor en la que operan varias técnicas para mejorar el aprendizaje del estudiante (.766), el grado de intervención que realiza el profesor al estudiante para trabajar con su personalidad (.835), el grado de la comodidad del estudiante con la enseñanza del profesor (.742). 
El grado de cumplimiento de las responsabilidades del profesor (.628), el nivel de mejora del estudiante en su rendimiento académico con la orientación del profesor (.709), el grado de desenvolvimiento del estudiante en las clases (.775), el grado de tener las mismas oportunidades que todos los estudiantes (.525). Por lo que se infiere que la oportunidad que tenga el estudiante para desarrollarse de manera positiva en el ámbito educativo depende de muchos aspectos ya que cada uno de ellos se debe sentir bien en el espacio en el que se rodea tanto en el ámbito educativo como el familiar, así al ver el estudiante que es apoyado, orienta y apreciado puede aumentar su motivación y confianza en si mismo para superarse y lograr los objetivos que se plantea.

\section{Discusión}

(Santodomingo, 2016), La Orientación Docente es el rol que puede llegar a desarrollar cualquier profesor de un centro educativo - deseablemente todos los docentes y a ser posible de manera coordinada con los demás profesores y con los orientadores - a través del cual proporcionará un apoyo a todo su alumnado para que pueda tomar decisiones sobre su itinerario formativo y su futuro profesional en las mejores condiciones posibles.

Se está de acuerdo con el autor en una parte ya que como menciona, la orientación docente es el rol que hace el profesor, pero nos solo para tomar decisiones en el itinerario formativo, si no para ayudar al estudiante con los problemas que tiene al momento de aprender y orientarlo para que el mejore.

Slideshare (2012), La estructura familiar es un conjunto en interacción, organizado de manera estable y estrecha en función de necesidades básicas con una historia y un código propio que le otorga singularidad.

Se está de acuerdo con la página ya que la estructura familiar es donde el estudiante se va desarrollando y donde él va cumpliendo sus necesidades básicas tanto como en el alimento o necesidades fisiológicas como en las necesidades educativas ya que es el primer lugar donde el estudiante va aprendiendo, también como en las necesidades emocionales, es decir la familia permita que el estudiante exprese sus emociones o sentimientos.
Silva., 2011), La estructura familiar es el soporte de todos los rasgos que caracterizan a una familia, le da identidad y distingue a una de otra. Esta identidad debe tener solidez y flexibilidad de límites.

Se está de acuerdo con la autora ya que como menciona la estructura familiar es la conformación que caracteriza a la familia, permite que se distingan todos sus aspectos que la conforman.

\section{Conclusión}

Se puede concluir que la familia es un factor importante en la orientación docente del estudiante ya que si el estudiante tiene relaciones familiares cercanas, donde ellos se sientan queridos y aceptados tienen un efecto positivo en el desempeño escolar de los estudiantes. Del mismo modo, las familias donde no hay violencia facilitan que los estudiantes aprendan y se desarrollen de manera adecuada.

También los estudiantes aprenden mejor cuando viven en familias que utilizan la disciplina de modo democrático, es decir, a los estudiantes se les explica las razones de las normas y en ocasiones también participan en las decisiones. Es muy importante que los adultos de la casa estén coordinados respecto a las normas, eso les brinda seguridad y confianza a los estudiantes, el sentir que ambos padres están de acuerdo les permite entender que es por el bien de ellos.

La buena comunicación en la familia es un aporte en la educación de los estudiantes. El estudiante se siente valioso e importante cuando sus padres lo escuchan, le preguntan y le dedican atención. Esto les permite tener mejores relaciones, aprenden ellos también a expresarse y escuchar a los demás, lo que los ayudará a lo largo de la vida en los desafíos académicos y en las relaciones personales. Para que un estudiante pueda desempeñarse correctamente con la orientación que le brindan sus docentes es importante que la familia siempre este con ellos y estén en un ambiente bueno con ellos, ya que ellos son los que afectan en su desempeño tanto en cómo está organizada su familia, como se apoya, en como cada integrante de la familia realiza un papel importante, el amor que se tienen, etc. Todos estos aspectos hacen que el estudiante mejore y se supere día con día en el ámbito educativo y en la vida social. 


\section{Referencias}

Maher, \& Forman. (1987). CONCEPTO DE EDUCACIÓN EDUCATIVA. Recuperado el 2 de Septiembre de 2020, de CONCEPTO DE EDUCACIÓN EDUCATIVA: https://rieoei.org/historico/deloslectores/736Mo lina108.PDF

Ortega, B. M. (2007). Estructura y función familiar. Recuperado el 18 de Septiembre de 2020, de Estructura y función familiar: https://www.sciencedirect.com/sdfe/pdf/downlo ad/eid/1-s2.0-S1134207207740185/first-pagepdf

Santodomingo, R. (12 de Octubre de 2016). Magisterio. Recuperado el 28 de Agosto de 2020, de Magisterio: https://www.magisnet.com/2016/10/laimportancia-del-profesor-orientador/

Silva., M. M. (6 de Noviembre de 2011). Slideshare. Recuperado el 28 de Agosto de 2020, de Silideshare: https://es.slideshare.net/monik73/estructurafamiliar10051423\#: :text=La\%20estructura\%20familia r\%20es\%20el,tener\%20solidez\%20y\%20flexibi lidad\%20del\%C3\%ADmites.

slideshare. (18 de Noviembre de 2012). Recuperado el 18 de Septiembre de 2020, de slideshare: https://es.slideshare.net/Psicoptolosas/estructura-familiar-15235234

Solórzano, M. d. (23 de Febrero de 2020). medicosfamiliares. Recuperado el 18 de Septiembre de 2020, de medicosfamiliares: https://www.medicosfamiliares.com/familia/elrol-en-la-familia-roles-familiares.html 